\title{
Search for photons at the Pierre Auger Observatory
}

\author{
M. Risse ${ }^{a *}$ for the Pierre Auger Collaboration \\ a Bergische Universität Wuppertal, 42119 Wuppertal, Germany
}

The Pierre Auger Observatory has a unique potential to search for ultra-high energy photons (above $\sim 1$ EeV). First experimental limits on photons were obtained during construction of the southern part of the Observatory. Remarkably, already these limits have proven useful to falsify proposals about the origin of cosmic rays, and to perform fundamental physics by constraining Lorentz violation. A final discovery of photons at the upper end of the electromagnetic spectrum is likely to impact various branches of physics and astronomy.

\section{Introduction}

There are good reasons to search for ultra-high energy (UHE, above $\sim 10^{18} \mathrm{eV}=1 \mathrm{EeV}$ ) photons at the Auger Observatory [1. An UHE photon produces a "normal" air shower, easily detectable with a giant shower observatory. Still, such photon showers have characteristics that make them well distinguishable from showers initiated by primary hadrons. The calculation of these photon shower characteristics can be done with high confidence because QED effects dominate, such that solid conclusions can be drawn from the data. A substantial flux of UHE photons is predicted in top-down models 2. Even in conventional cosmic-ray scenarios, UHE photons may be produced at detectable level [3]; as their expected flux at Earth depends on uncertain parameters such as source density, injection spectrum or extragalactic radio background, findings on UHE photons will provide valuable astrophysics information.

In the following, the current status of the search for UHE photons at the Pierre Auger Observatory is briefly summarized. Detailed descriptions of the data analyses can be found in Refs. [415. A general review on the search for UHE photons using air showers is given in Ref. [6].

\section{Search with hybrid data}

Showers initiated by UHE photons develop differently from showers induced by nuclear primaries. Particularly, observables related to the development stage or "age" of a shower (such as the depth of shower maximum $X_{\max }$ ) and to the content of shower muons provide good sensitivity to identify primary photons. Photon showers are expected to develop deeper in the atmosphere (larger $X_{\max }$ ), see Fig. 1. This is connected to the smaller multiplicity in electromagnetic interactions compared to hadronic ones, such that a larger number of interactions is required to degrade the energy to the critical energy where the cascading process stops. Additionally, the LPM effect [7] results in a suppression of the pair production and bremsstrahlung cross-sections. Photon showers also contain fewer secondary muons, since photoproduction and direct muon pair production are expected to play only a sub-dominant role.

Using high-quality hybrid events (air showers registered by both the fluorescence telescopes and the ground array which fullfil certain strict requirements to be accepted for the analysis), it is possible to compare directly the observed $X_{\max }$ values of UHE events with expectations for photon primaries of same energy and arrival direction. An example of a measured air shower and the comparison of its $X_{\max }$ value to results from photon simulations is shown in Fig. 2, One can

${ }^{*}$ Currently at University of Siegen, Germany 


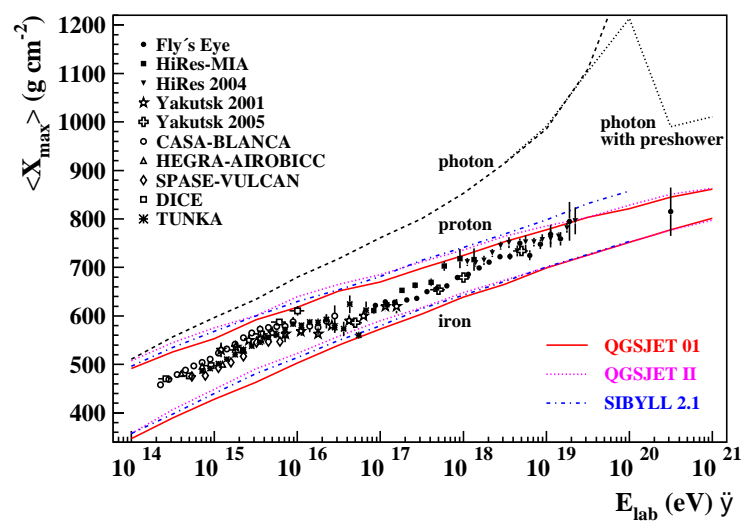

Figure 1. Average depth of shower maximum $<X_{\max }>$ versus energy simulated for primary photons, protons and iron nuclei. Depending on the specific particle trajectory through the geomagnetic field, photons above a few times $10^{19} \mathrm{eV}$ can also create a preshower (see e.g. Ref. [8] and references therein): as indicated by the splitting of the photon line, the average $X_{\max }$ values then do not only depend on primary energy but also arrival direction. For nuclear primaries, calculations for different hadronic interaction models are displayed. One sees that at $10 \mathrm{EeV}$, photons penetrate deeper into the atmosphere than hadron primaries by (on average) $\sim 200 \mathrm{~g} \mathrm{~cm}^{-2}$ or more. This is to be compared with the resolution of fluorescence telescopes of typically $\sim 20-30 \mathrm{~g} \mathrm{~cm}^{-2}$. (Figure taken from Ref. [4.)

see that the observed $X_{\max }$ is well below the values assuming primary photons. It is not likely that this air shower was initiated by an UHE photon.

Inspecting 29 such high-quality air showers with energies above $10 \mathrm{EeV}$, it turned out that in all cases the photon predictions exceed by far the observed $X_{\max }$ values. From this, an upper limit of $16 \%$ (95\% c.l.) on the cosmic-ray photon fraction could be obtained as published in 2007 4. Despite the very small number of events, this limit was the best one at that time above $10 \mathrm{EeV}$ (to be superseded by the limit us-
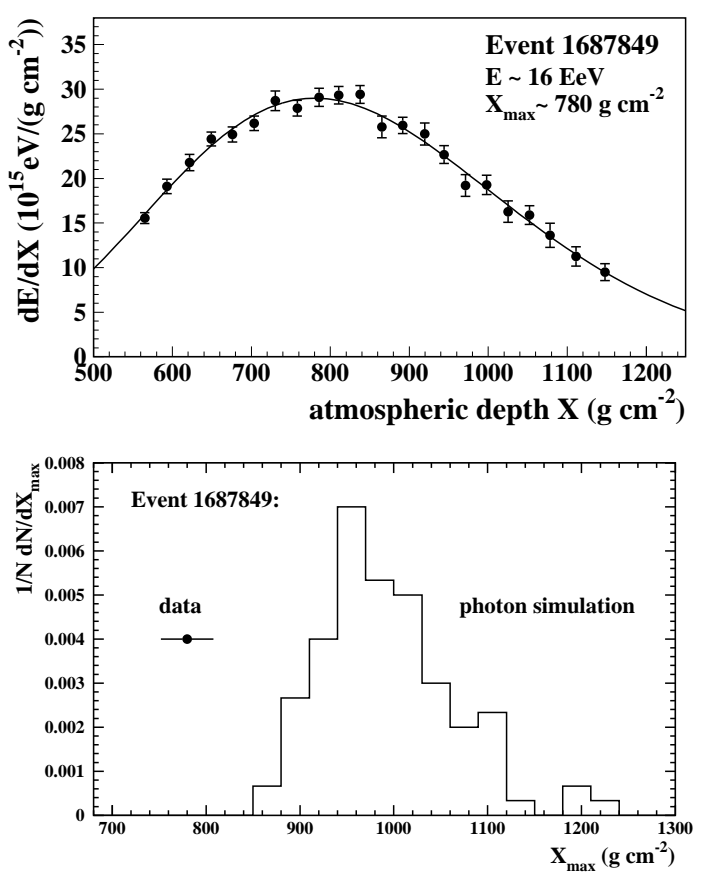

Figure 2. (Top) Example of a reconstructed longitudinal energy deposit profile (points) and the fit by a Gaisser-Hillas function (line). (Bottom) $X_{\max }$ measured in the shower shown in the top plot (point with error bar) compared to the $X_{\max }^{\gamma}$ distribution expected for photon showers (solid line). The observed $X_{\max }$ is well below the values assuming primary photons. (Figures taken from Ref. [4.)

ing Auger ground array data, see below). Moreover, the Auger hybrid data set was the first (and so far only) one where the fluorescence technique with its direct observation of $X_{\max }$ was applied to search for UHE photons.

\section{Search with ground array data}

To exploit the larger number of UHE events recorded by the ground array only (i.e. without requiring additional fluorescence telescope data), two observables of the array detectors were cho- 
sen in an analysis published in 2008 [5] which have significantly different behavior for nuclear primaries when compared to photons: the risetime of the recorded shower signal and the radius of curvature of the shower front (see Fig. 3 ). As an energy estimator, the total signal deposit $S(1000)$ derived for a ground detector at $1000 \mathrm{~m}$ core distance is used by comparing the measured value to photon shower simulations.

In Fig. 4, for each measured shower a quantity is plotted as a function of energy of the event that combines the discriminating observables of risetime and radius of curvature. Also shown are expectations from photon shower simulations. An $a$ priori cut had been defined such that data points lying above the mean of the photon distribution are considered as photon candidates. Accounting for inefficiencies, upper limits on the presence of photons in the primary cosmic-ray beam were placed; in particular a limit of $2 \%$ (at 95\% c.l.) above $10 \mathrm{EeV}$ was derived (see Fig. 5, lower plot).

Also, in this analysis the first direct limit to the flux of UHE photons (instead of the fraction) was obtained (Fig. 5, upper plot). In case of ground arrays, the flux (or a limit on the flux) is the more robust experimental quantity (see Refs. [56]).

\section{Implications}

As can be seen from Fig. 5, particularly from the comparison of experimental UHE photon flux limits and model predictions, contemporary top-down models are severely constrained now: the non-observation of UHE photons by the Auger Observatory is difficult to explain in exotic physics scenarios (see e.g. also Refs. 910]).

The photon limits also reduce systematic uncertainties in the derivation of the total cosmicray flux spectrum [11, since the energies of photon showers could be misreconstructed. As can also be seen in Fig. 4 there are no indications of an increasing photon component towards highest energies at the present sensitivity level.

The photon bounds have also proven useful for fundamental physics. In Ref. [12, the fraction of GZK photons expected at Earth was computed assuming that Lorentz invariance violation suppresses pair production by UHE photons. Values were found that exceed the Auger photon limits by about a factor 10. Corresponding constraints on Lorentz violation parameters improve previous constraints by several orders of magnitude due to the extreme energy in case of UHE photons.

\section{Outlook}

Data taken during the first phase of construction of the southern Auger Observatory allowed the derivation of UHE photons limits that approach (in terms of fraction) the $10^{-2}$ level at energies above $10 \mathrm{EeV}$. Both detector components (telescopes and array) offer excellent discrimination power between photon and hadron primaries such that the sensitivity level will continue to improve over the next years while the data accumulate. The upper range of predictions for GZK photons (see Fig. 5) as well as the yet unexplored energy range below $10 \mathrm{EeV}$ can be tested soon.

With a main goal of full sky coverage, the Auger Observatory is to be completed by a northern site. Current plans aim at a significantly (factor $\sim 7$ ) larger array to proceed with cosmic-ray astronomy. With such an enlarged northern site, photon fractions at or below the $10^{-3}$ level are in reach within few years of operation.

As for any exploratory search, the timescale for the final discovery of UHE photons is uncertain. However, even the very first results from the photon search at the Auger Observatory proved very helpful for cosmic-ray physics (e.g. discrimination between different cosmic-ray source scenarios) and for fundamental physics (test of Lorentz invariance). It seems reasonable to believe that this ability of the UHE photon search to provide substantial physics results will persist. The unprecedented and presently unique sensitivity of the Auger Observatory to UHE photons, particularly when completed by the northern site, allows for the first time a realistic search for UHE photons also from conventional cosmic-ray scenarios.

A final discovery of UHE photons would open a new - and the most extreme - "window" of photon astronomy. Experience shows that this is usually accompanied with radically new, and often unexpected, insights about the bizarre inhabitants of the universe and their (inter-)actions 13 
(for a surely incomplete list of possible impacts of a discovery of UHE photons on various research fields, see Ref. [6]).

Acknowledgments. It is a pleasure to thank the CRIS organizers for an excellent and inspiring conference. (The sometimes quite "German" weather made me feel at home even more.) Partial support from the German Ministry for Education and Research BMBF (Verbundforschung Astroteilchenphysik) and from the DFG are kindly acknowledged.

\section{REFERENCES}

1. J. Abraham et al. (Pierre Auger Collaboration), Nucl. Instrum. Meth. A 523, 50 (2004).

2. P. Bhattacharjee, G. Sigl, Phys. Rep. 327, 109 (2000).

3. G. Gelmini, O. Kalashev, D.V. Semikoz, [arXiv:astro-ph/0706.2181]; G. Sigl, Phys. Rev. D 75, $103001 \quad$ (2007) arXiv:astro-ph/0703403.

4. J. Abraham et al. (Pierre Auger Collaboration), Astropart. Phys. 27 (2007) 155

5. J. Abraham et al. (Pierre Auger Collaboration), Astropart. Phys. 29 (2008) 243

6. M. Risse, P. Homola, Mod. Phys. Lett. A 22, 749 (2007).

7. L.D. Landau, I.Ya. Pomeranchuk, Dokl. Akad. Nauk SSSR 92, 535 \& 735 (1953);

A.B. Migdal, Phys. Rev. 103, 1811 (1956).

8. P. Homola et al., Comp. Phys. Comm. 173, 71 (2005)

9. R. Aloisio, F. Tortorici, Astropart. Phys. 29, 307 (2008).

10. M. Kachelrieß, arXiv:0810.3017v2

11. Pierre Auger Collaboration, Phys. Rev. Lett. 101, 061101 (2008)

12. M. Galaverni, G. Sigl, Phys. Rev. Lett. 100, 021102 (2008).

13. A. Lawrence, arXiv:0704.0809
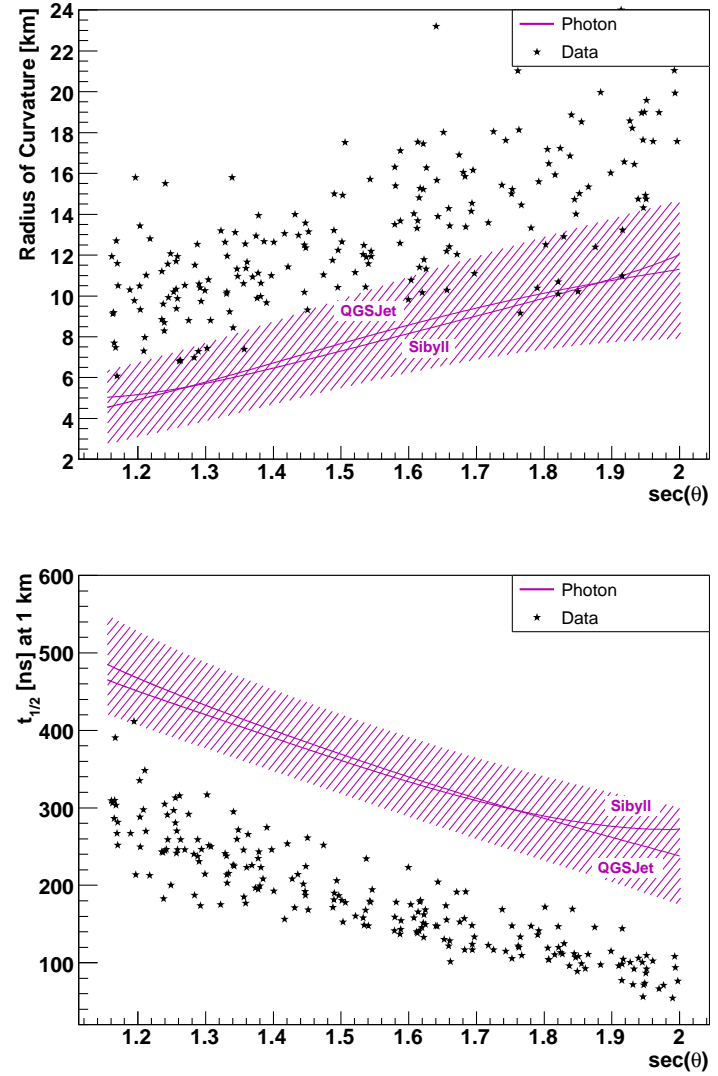

Figure 3. Parameterization of the mean behavior of the radius of curvature $R$ (upper plot) and shower risetime at $1000 \mathrm{~m}$ core distance $t_{1 / 2}$ (lower plot) for $20 \mathrm{EeV}$ primary photons as a function of the zenith angle using two different hadronic interaction models. An increase (a decrease) of $R$ (of $t_{1 / 2}$ ) with zenith angle is expected due to the generally longer path lengths to ground in case of larger inclination. Real events of 19-21 EeV (photon energy scale) are added. The significant deviation of the observed values from those expected for primary photons is visible. (Figures taken from Ref. [5].) 


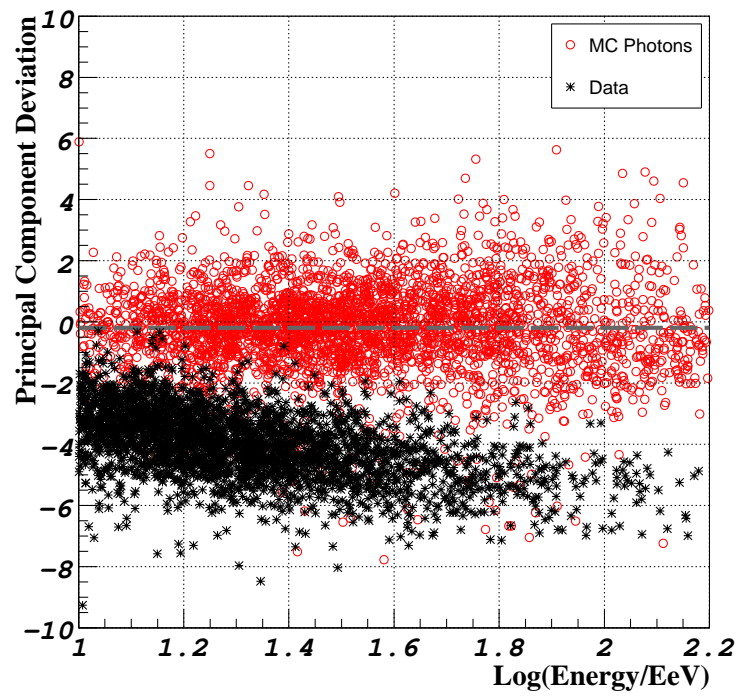

Figure 4. Plotted is a quantity that combines the measurements of radius of curvature and shower risetime (cf. Fig. 3) for data (black crosses) and photon simulations (open red circles) as a function of the primary energy (photon energy scale). Data lying above the dashed line, which indicates the mean of the distribution for photons, are taken as photon candidates. No event meets this requirement. Moreover, no trend is visible of higher-energy events becoming more photon-like. (Figure taken from Ref. [5].)
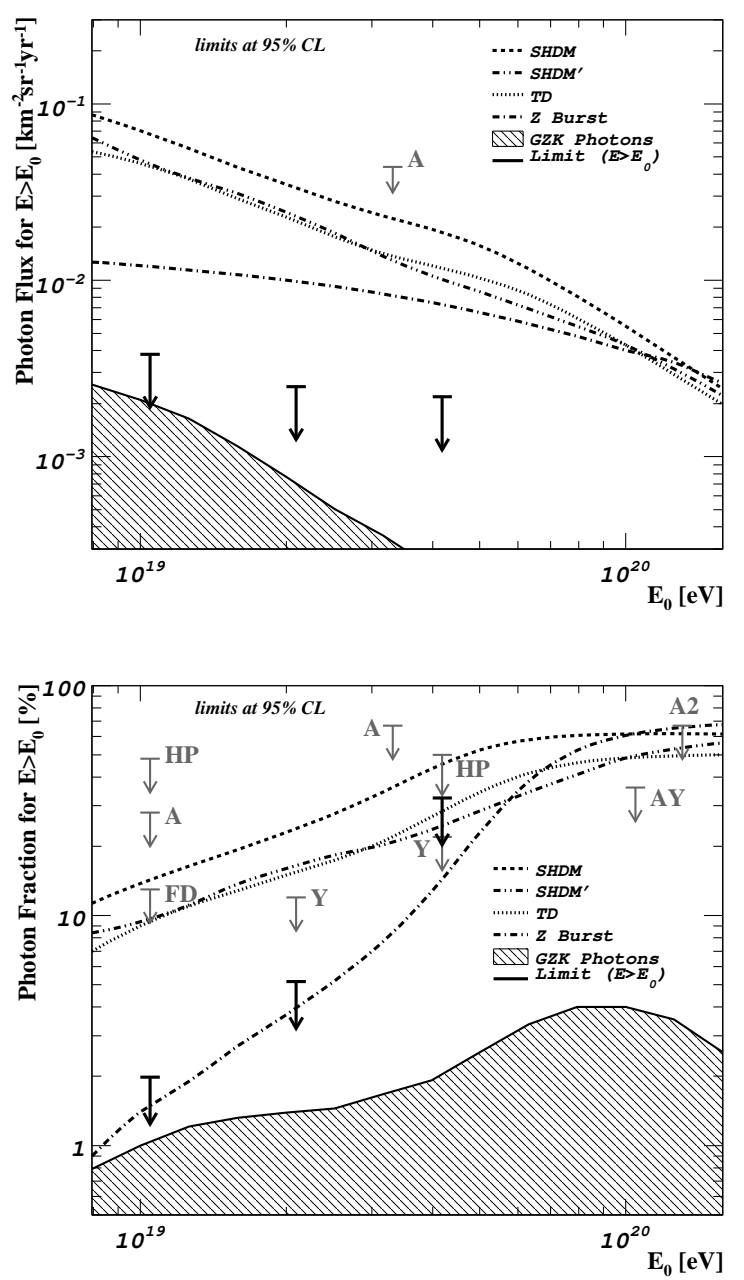

Figure 5. The upper limits on the integral flux of photons (top) and on the fraction of photons in the integral cosmic-ray flux (bottom) as a function of the threshold energy as measured by the Auger array detector (black arrows) along with previous experimental limits and model predictions. (Figures taken from Ref. [5].) 